\title{
Geografías de la desposesión en la ciudad neoliberal: ejecuciones hipotecarias y vulnerabilidad social en Santa Cruz de Tenerife (Canarias-España)
}

Juan-Samuel García-Hernández. Universidad de La Laguna, San Cristóbal de La Laguna, España.

Carmen Ginés-De la Nuez. Universidad de Las Palmas de Gran Canaria, Las Palmas de Gran Canaria, España.

RESUMEN | En el contexto general de los procesos de acumulación por desposesión, se examinan las lógicas socioespaciales de los desalojos por ejecución hipotecaria y su incidencia en la extensión de la vulnerabilidad social en Santa Cruz de Tenerife. El análisis contempla, por un lado, los vínculos entre la expansión inmobiliaria anterior a la crisis y el reparto intraurbano de las ejecuciones; y, por otro, mediante el análisis de caso, su espacialidad y significado en un barrio socialmente vulnerable. La combinación de métodos permite estudiar tanto la intensidad y distribución de los desalojos en las distintas áreas sociales, como la extensión de la vulnerabilidad que entraña la pérdida de la vivienda. Los resultados muestran cómo las ejecuciones han contribuido a la difusión espacial de la vulnerabilidad social y revelan, a la vez, las dificultades a las que se enfrentan, en su vida cotidiana, los residentes en los espacios afectados por tales procesos.

PALABRAS CLAVE | transformaciones socioterritoriales, política habitacional, periferia urbana.

ABSTRACT | In the general context of the processes of accumulation by dispossession, the socio-spatial logics of evictions due to foreclosure and their incidence on the extent of social vulnerability in Santa Cruz de Tenerife, are examined. The analysis contemplates, on the one hand, the links between the real estate expansion prior to the crisis and the intra-urban distribution of mortgage foreclosures and, on the other, through case analysis, its spatiality and meaning in a socially vulnerable neighborhood. The combination of methods allows the analysis of the intensity and distribution of the evictions in the different social areas and the extension of the vulnerability that the loss of the housing entails. Results show how foreclosures have contributed to the spatial diffusion of social vulnerability and reveal, at the same time, the difficulties faced by residents in the spaces affected by such processes.

KEYWORDS | socio-territorial transformations, housing policy, urban periphery.

Recibido el 21 de junio de 2018, aprobado el 8 de abril de 2019.

E-mails: J.-S. García-Hernández, jgarciah@ull.edu.es |C. Ginés-de la Nuez, carmen.gines@ulpgc.es 


\section{Introducción}

En paralelo con el repliegue del capitalismo industrial, desde las últimas décadas del siglo xx se consolida el auge del sector financiero-inmobiliario, que abandona su carácter de accesorio del circuito convencional y se convierte en el motor principal de la dinamización de la economía mundial (Charnock, Purcell, \& Ribera-Fumaz, 2015; Coq-Huelva, 2013; Harvey, 2007). El crecimiento urbanístico, acompañado de leyes propicias y falta de control político, es uno de los pilares que garantizan la circulación de capital al aumentar la plusvalía sustentada en la especulación inmobiliaria (García, 2010; Lois, Piñeira, \& Vives, 2016; López \& Rodríguez, 2011). La ocupación desmedida de suelo para la expansión de la superficie residencial deriva en una serie de problemas urbanísticos, medioambientales, paisajísticos y económicos que han sido señalados por la teoría urbana crítica (Brenner \& Theodore, 2002). Los planteamientos desarrollados afirman que la orientación de los excedentes de capital hacia el sector inmobiliario se halla en la raíz de las crisis que afectan periódicamente al sistema (Harvey, 2003).

En España, entre 1997 y 2006 se produjo un boom inmobiliario de envergadura, con unos niveles de construcción de viviendas que superaron, con mucho, el incremento en el número de hogares (Fernández Durán, 2006; López \& Rodríguez, 2011). La permisividad de la legislación estatal y la orientación de las políticas públicas regionales y locales resultan clave en la comprensión del proceso. La Ley 6/1998 determina que podría ser urbanizable todo el suelo rústico, salvo aquel con un valor singular necesario de proteger (Burriel, 2008), lo que facilita que las actuaciones de los gobiernos se plieguen a los intereses de los agentes privados. Tal es así que, al tiempo que se proporciona capacidad de decisión y de acción a los grupos privilegiados, se renuncia a salvaguardar una serie de necesidades colectivas en las que se fundamenta el derecho a la ciudad (Lefebvre, 1969). La producción de vivienda queda liberada de la interferencia de las administraciones públicas, produciéndose, por tanto, una deslegitimación del planeamiento como instrumento de control (Burriel, 2008) y de justicia socioespacial. El resultado es una expansión carente de regulación que se ha traducido en la artificialización indiscriminada del suelo (Lois, Piñeira, \& Vives, 2016), sustentada en una economía altamente financiarizada cuyos efectos perversos salen a la luz con la crisis hipotecaria. Se reconoce, por tanto, que con el estallido de la burbuja inmobiliaria afloraron diversos problemas sociales gestados en la fase previa, y que dibujan un ciclo completo de crecimiento y desplome propio de las prácticas capitalistas de acumulación por desposesión (Harvey, 2007). La evidencia de tales estrategias ha sido la privación de la vivienda a través de las ejecuciones hipotecarias, una situación especialmente grave en el caso espańol, por la falta de mecanismos legales que liberen de la deuda a personas que se hipotecaron en un momento de escasez de vivienda asequible y concesión irresponsable de créditos hipotecarios (Human Rights Watch, 2014).

Este estudio examina las lógicas socioespaciales de la desposesión de vivienda en una capital insular del archipiélago canario, Santa Cruz de Tenerife. En primer lugar, se analiza la desigual magnitud de las ejecuciones hipotecarias en las diferentes áreas sociourbanas, a partir de la consideración de que la fase de expansión inmobiliaria 
condiciona la espacialidad de los problemas que se desencadenan con la crisis hipotecaria. Se persigue comprobar si las ejecuciones muestran una mayor intensidad en las áreas de habitación de la clase trabajadora, al igual que ocurre en otras realidades geográficas (Aalbers, 2009; Gutiérrez \& Declòs, 2017; Vives-Miró, González, \& Rullán, 2015). En segundo lugar, se aborda la incidencia de estos procesos en un barrio vulnerable en el que se registra un número destacado de desalojos. El estudio de caso permite comprobar, a escala de calle y número, la distribución espacial de las ejecuciones hipotecarias, al tiempo que se sondean las prácticas sociales de los residentes en un barrio afectado por la pérdida de la vivienda. Este modo de proceder pretende conocer las reacciones y comportamientos de los vecinos, que conforman una red de relaciones que le otorgan sentido y diferencia al lugar (Carlos, 2007). Tal es así, que en las esferas de la vida cotidiana resulta posible identificar una producción social de la ciudad, inserta en el marco de un espacio percibido y vivido del que los residentes son partícipes y protagonistas (Lefebvre, 1974). Se explora la hipótesis de que en los lugares de alojamiento de clase trabajadora gestados al amparo del urbanismo neoliberal (Bauman, 2001; Brenner \& Theodore, 2002; Hackworth, 2013), la debilidad de los vínculos vecinales y la ausencia de estrategias colectivas para la superación de las dificultades contribuyen a la intensificación de su vulnerabilidad.

El interés de la investigación estriba en analizar las geografías de la crisis hipotecaria a una escala intraurbana desde una doble vertiente: en primer lugar, un análisis cuantitativo que muestre las lógicas que imperan en la espacialidad del fenómeno; $y$, en segundo lugar, un acercamiento cualitativo que tantee las prácticas de los residentes en un barrio en el que la pérdida de la vivienda es un problema patente. El archipiélago canario es un territorio donde se precisa el estudio de tales procesos, pues, al igual que en el litoral mediterráneo y en las grandes áreas metropolitanas, los excesos de su proceso urbanizador con fines especulativos explican la intensidad de la crisis inmobiliaria y la magnitud de las ejecuciones hipotecarias a partir de 2007 (Gutiérrez \& Domènech, 2017; Méndez \& Plaza, 2016; Obeso, 2014). En Tenerife, el impacto de los desalojos fue notable en aquellas áreas insulares con dinámicas inmobiliarias desorbitadas $y$, entre estas, se vieron especialmente afectadas las destinadas al alojamiento de población trabajadora (García-Hernández, DíazRodríguez, \& García-Herrera, 2018). Las instituciones financieras y los fondos de capital privado fueron los artífices principales de la acumulación y se convirtieron, tras la crisis, en los principales beneficiarios de la desposesión (Vives-Miró \& Gutiérrez, 2017). En las islas, parte importante de las exenciones fiscales estuvo dirigida al sector inmobiliario y alentó la inversión extranjera en las principales áreas urbanas y turísticas (García-Herrera, Smith, \& Mejías, 2007).

En la ciudad de estudio, Santa Cruz de Tenerife, existen indicios de la adopción, por parte de los gobiernos locales, de políticas urbanas de sesgo neoliberal. Apuntan en esa dirección las transformaciones recientes de las áreas centrales y una planificación pública marcada por los intereses de los agentes privados (Armas, 2016). En un marco de creciente competitividad urbana, se ha señalado que el empeńo por la promoción de la ciudad se traduce en la concentración de inversiones en sectores concretos y en la paulatina desatención de las periferias (Díaz 
et al., 2002; García-Hernández, 2017; García-Herrera et al., 2007). Con la crisis de 2007, se asiste al empeoramiento de las condiciones de vida en la ciudad y, en particular, en los espacios urbanos previamente vulnerables que se enfrentan al deterioro de sus indicadores socioeconómicos -desempleo, precariedad laboral, solicitantes de asistencia social, etcétera- (Instituto Municipal de Atención Social [IMAs], 2014). En ese contexto temporal, el reparto espacial de las ejecuciones hipotecarias podría introducir matices reseñables para la redefinición de la geografía social urbana, tal y como se ha constatado en otros escenarios (Gutiérrez \& Declòs, 2017; Parreño-Castellano, Domínguez-Mujica, Armengol-Martín, Pérez, \& Boldú, 2018; Vives-Miró, Rullán, \& González, 2018). El planteamiento teórico de que las crisis capitalistas contribuyen al agravamiento del desarrollo urbano desigual (Smith, 2010) puede ser cotejado a partir del examen de la densidad de los desalojos en los barrios que conforman la ciudad; mientras que el estudio de caso resulta significativo para comprender la espacialidad de la desposesión de vivienda a una escala local e identificar su incidencia en la difusión de la vulnerabilidad social. En tal sentido, resulta paradigmático el caso de Añaza, un barrio que la administración promovió en la década de 1980 para el alojamiento de grupos desfavorecidos y que registró, durante la etapa de urbanismo expansivo, un destacado crecimiento inmobiliario que explica que los desalojos adquirieran mayor entidad que en los barrios socialmente vulnerables originados y colmatados con anterioridad.

El trabajo se organiza en cinco apartados: en el primero se presenta el escenario de endeudamiento y desposesión que enmarca los procesos que se analizan; el segundo recoge las fuentes y la metodología; el tercero delimita y caracteriza el ámbito de estudio; en el cuarto y quinto se plantean los resultados de la investigación y, por último, se apuntan unas reflexiones finales.

\section{Los mecanismos de acumulación del neoliberalismo: del endeudamiento a la desposesión}

El vínculo entre la urbanización y los procesos de acumulación de capital se ha puesto de relieve a lo largo de la historia del capitalismo, considerándose a la primera un ingrediente básico de la supervivencia del sistema y objeto crucial de la lucha de clases (Harvey, 2007; Lefebvre, 1974). Con el neoliberalismo, la especialización en el circuito secundario implica una expansión inmobiliaria sin precedentes, que reafirma el papel central de la ciudad en la absorción de los excedentes de capital (Coq-Huelva, 2013; Lois, Piñeira, \& Vives, 2016; López \& Rodríguez, 2011). El proceso de mercantilización del espacio urbano vinculado a la producción masiva de vivienda se hizo factible con la expansión del crédito hipotecario y el endeudamiento familiar. La titulización hipotecaria se convirtió en protagonista de la reproducción ampliada del capital y fue un componente fundamental de un mecanismo de cercamiento de la vivienda, confirmado con la oleada de desahucios que tuvo lugar bajo el régimen de acumulación por desposesión que se estableció a partir de la crisis (Aalbers, 2009; Gutiérrez \& Vives-Miró, 2018). Por tanto, aunque la apropiación de la renta urbana a partir de la adquisición de títulos inmobiliarios-financieros pueda ser considerada uno de los factores esenciales del último ciclo alcista, no es 
menos cierto que también debe ser identificada como uno de los principales detonantes de la crisis actual (Vives-Miró, González, \& Rullán, 2015). De este modo, la premisa de que la acumulación por desposesión es un rasgo inherente a las lógicas de funcionamiento del sistema capitalista queda revalidada en el marco del urbanismo neoliberal (Harvey, 2003). La financiarización del entorno construido y, en particular, de la vivienda, lo corrobora, pues el endeudamiento de las familias en un contexto de desregulación desemboca, a partir de 2007, en un volumen de desalojos sin precedentes que constituyen un reflejo de los procesos de desposesión ligados a las crisis capitalistas (Gutiérrez \& Declòs, 2017). Por tanto, resulta pertinente comprender que la crisis financiera no es simplemente la proliferación fortuita de incumplimientos y ejecuciones hipotecarias. Se han sucedido diversas estrategias deliberadas de canalización de los capitales hacia el circuito secundario (Burriel, 2008; Naredo, 2010; Vinuesa, 2013), que incentivaron unos niveles de producción de viviendas superiores a las necesidades habitacionales, primando la búsqueda de la rentabilidad económica a corto plazo sobre la inexcusable función social de la vivienda. Tal como indica Aalbers (2009), un ingrediente clave de la crisis fue la generalización de préstamos de alto riesgo en condiciones abusivas que atraparon a los prestatarios más vulnerables. Las hipotecas permitían al ciudadano financiar el valor total del inmueble, y a veces más, incorporando otros bienes a la deuda casi al mismo coste que un alquiler. La destrucción masiva de empleo, el deterioro de las condiciones de vida y trabajo y la precariedad laboral estuvieron estrechamente relacionados con la generalización de las ejecuciones hipotecarias, afectando así en mayor proporción a los grupos sociales menos favorecidos (Gutiérrez \& Domènech, 2017). Además, como consecuencia de los desalojos, los bancos han emergido como grandes tenedores de vivienda y, a la espera de posibles revalorizaciones futuras, serán actores clave en la próxima fase alcista del mercado de vivienda (Gutiérrez \& Domènech, 2018).

Un aspecto esencial es la comprensión de la territorialidad del fenómeno, pues la elección de determinadas áreas para la inversión en el circuito secundario, y la permisividad de las administraciones y las leyes, repercutieron en el nivel de endeudamiento durante la expansión inmobiliaria y en la desigual distribución de los impagos en el periodo de crisis (Burriel, 2016). Tal es así, que se reconoce que la sobredimensión que alcanzó en España la producción de vivienda hizo que el pinchazo de la burbuja fuera más traumático que en otros países europeos (Naredo, 2010). A una escala regional se comprueba, también, la mayor vulnerabilidad de aquellos territorios que se especializan en el negocio turístico-inmobiliario (GarcíaHernández, Díaz-Rodríguez, \& García-Herrera, 2018; Méndez \& Plaza, 2016; Obeso, 2014); por su parte, a una escala urbana -según señalan Piñeira y Trillo (2016)-, las grandes ciudades, dadas sus economías más diversificadas, estarían en mejores condiciones de afrontar las crisis que las ciudades pequeñas y de tamaño medio, por lo general dependientes de sectores en los que la recesión ha impactado con dureza. Finalmente, el análisis intraurbano permite cotejar la dimensión de clase de la crisis hipotecaria, pues esta muestra una mayor incidencia en las áreas de residencia de población de bajos ingresos (Vives-Miró, Rullán, \& González, 2018). A esta escala, se persigue comprender las lógicas de distribución más concretas de 
las ejecuciones y aproximarse a las geografías de la desposesión desde una perspectiva basada en sus manifestaciones cotidianas. El crecimiento urbanístico neoliberal habría reforzado la polarización del espacio urbano al producir conjuntos residenciales dirigidos a la población trabajadora, a la que se facilita el acceso a una vivienda mediante el crédito hipotecario. Se consolida una urbanización difusa e incontrolada que, siguiendo la estela del precio del suelo más barato, deriva en el desarrollo de piezas urbanas aisladas que precisan de grandes infraestructuras de transporte para su conexión (Lois, Piñeira, \& Vives, 2016). El resultado es la creación de nuevas periferias que se extienden por las coronas metropolitanas, conformando espacios urbanos de gran homogeneidad edificatoria e importante huella ecológica y paisajística y donde predomina la vivienda hipotecada, cuyos residentes mantienen frágiles lazos de amistad y sentimientos de pertenencia (Bauman, 2001; Naredo, 2010; Theodore, Peck, \& Brenner, 2009). Se gestan de este modo áreas cada vez más segregadas en las que la idea de ciudad como proyecto colectivo se desvanece, y que se convierten en escenarios ańadidos de vulnerabilidad social.

\section{Fuentes y apuntes metodológicos}

El estudio de las geografías de la desposesión en la ciudad de Santa Cruz de Tenerife se aborda a través de procedimientos distintos pero complementarios. El uso de una metodología mixta contribuye a enriquecer el resultado de la investigación social (Bülher, Kaspar, \& Ostermann, 2010; Iveson, 2007), en particular en las áreas urbanas, pues -como sostiene Lefebvre (1969)- la comprensión de los contenidos y significados que aglutina la ciudad requiere diferentes perspectivas de análisis y no un solo modo de operar. Las técnicas utilizadas en este trabajo aportan un conocimiento sobre la realidad sociourbana desde distintos ángulos y proporcionan respuesta a las preguntas de investigación (Winchester \& Rofe, 2010).

En primer lugar, el análisis documental permite caracterizar el crecimiento inmobiliario y contextualizar la situación socioeconómica de la ciudad y el barrio de estudio. Se han consultado los informes del Ministerio de Vivienda sobre la burbuja de precios y la producción de vivienda entre 1997 y 2007 (Arcas, Muñoz, \& Cristóbal, 2007); el Atlas de la Vulnerabilidad Urbana de Espańa de 2011, el Censo de Población y Viviendas de 2011, el informe del Observatorio Socioeconómico de la Sociedad de Desarrollo de 2013 y el Diagnóstico Social del imas de 2014, que ofrecen datos con cierto nivel de detalle sobre la situación social de la ciudad de estudio; mientras; el Plan General de Ordenación de 2013 y las fotografías aéreas verticales y ortofotos permiten identificar, respectivamente, la tipología de las viviendas -libre y protegida- y las etapas de crecimiento del barrio de Añaza, para su vinculación posterior con las ejecuciones hipotecarias.

En segundo lugar, se procede a la explotación de una fuente de información inédita, la base de datos Atlante del Consejo General del Poder Judicial (cGPJ), que proporciona el domicilio de notificación de los procedimientos judiciales que por impago de la hipoteca o el alquiler se iniciaron, entre 1999 y 2016, en los distintos juzgados de primera instancia e instrucción de Canarias. Esta información permite, pues, analizar la evolución anual de los desalojos y -lo que es más importante- su 
modalidad y distribución intraurbana a escala de calle y número. El procesado de la información consistió en la desagregación de los registros por provincias, islas y municipios, para obtener los correspondientes al término de Santa Cruz de Tenerife; a continuación, se procedió al tratamiento de la información y a la georreferenciación de las ejecuciones para su encaje automático con la cartografía catastral; y, por último, se asoció cada uno de los registros a los barrios de la ciudad y, en el caso de Añaza, a los distintos bloques a partir de su dirección postal. Finalmente, se procedió a la obtención de las tasas de ejecuciones hipotecarias por cada mil habitantes y a su representación cartográfica.

Por último, la investigación cualitativa dirigió su atención a descubrir las experiencias de los residentes en Añaza, un barrio vulnerable desde su origen donde los desalojos por ejecución hipotecaria alcanzan un importante volumen. Para aproximarnos a las prácticas de los residentes en este barrio, se optó por la realización de entrevistas semiestructuradas en profundidad, una modalidad idónea para recolectar información sobre comportamientos, motivaciones, opiniones o significados (Hay, 2010; Taylor \& Bogdan, 1996). El conocimiento de la experiencia individual de las personas posee interés en la medida en que los relatos no solamente informan de sus características personales, sino que revelan aspectos de su posición en la estructura social (Winchester $\&$ Rofe, 2010) y constituyen, por tanto, una vía para la comprensión de las relaciones de dominación propias del escenario urbano neoliberal (Brenner \& Theodore, 2002; Hackworth, 2013). Se efectuó un total de quince entrevistas repartidas entre residentes de diferentes perfiles -género, edad, ubicación en viviendas protegidas y libres del barrio, grado de implicación en colectivos vecinales-. Las preguntas se dirigieron a captar aspectos que resultaran indicativos de sus condiciones de existencia cotidiana -situación social, estado de sus viviendas, implicación en colectivos y actividades del barrio, sentimientos de pertenencia y vínculo social, etcétera-. Las conversaciones fueron grabadas y posteriormente transcritas para analizar su contenido y extraer los significados más relevantes. Se siguió la estrategia propuesta por Mayring (2000) que, tras clasificar las alocuciones en unas categorías de análisis, privilegia los fines más interpretativos del procedimiento y reduce la atención habitual a la cuantificación de la información discursiva.

\section{Contexto espacial: la ciudad de Santa Cruz y el barrio de Ańaza}

Santa Cruz de Tenerife es una ciudad con una clara diferenciación socioespacial. La producción inmobiliaria en la fase expansiva del ciclo muestra un comportamiento geográfico dispar, que resulta esencial para interpretar la magnitud de los problemas que emergieron al desplomarse el mercado. En la ciudad se pueden diferenciar tres sectores: el centro urbano y las dos periferias obreras (figura 1). El centro urbano incluye los espacios emblemáticos de la ciudad y está constituido por los lugares habituales de alojamiento de las clases medias y altas: la ciudad histórica y sus ensanches, sectores de ciudad jardín y áreas de nueva centralidad resultado de la gentrificación de barrios populares a finales del siglo xx. La primera periferia está integrada por un amplio conjunto de barrios de clase obrera surgidos desde mediados del 
siglo xx y con reducida actividad inmobiliaria desde la década de 1980. Se trata de un conjunto heterogéneo con protagonismo de la autoconstrucción y en el que se inserta buena parte de la vivienda pública de la ciudad y promociones de vivienda privada previas a la expansión de la fase neoliberal. Finalmente, la segunda periferia es un sector más alejado que se desarrolló a partir de 1975 para el alojamiento de población de bajos ingresos y que ha concentrado, por tanto, la mayor parte de la expansión inmobiliaria reciente de la ciudad, con viviendas unifamiliares adosadas y promociones de vivienda pública, protegida y privada en bloques de altitud media y elevado número de viviendas (Díaz et al., 2002).

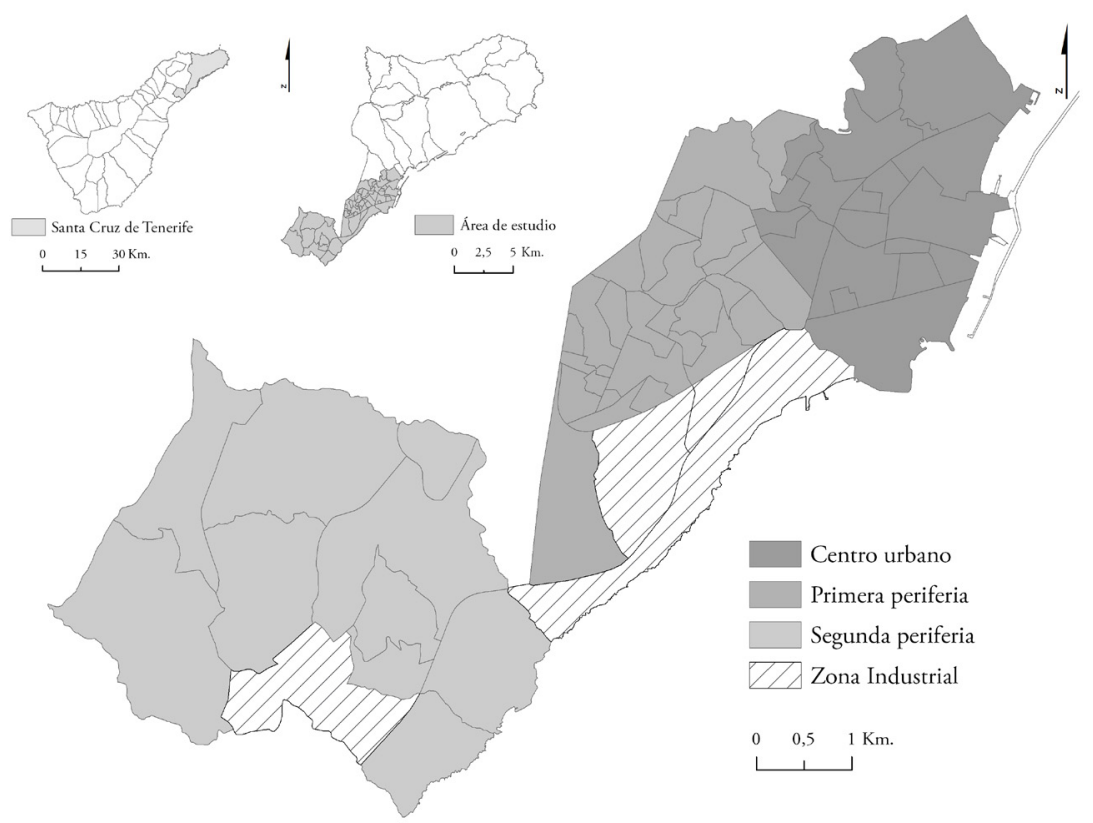

Figura I | Principales áreas urbanas de la ciudad de Santa Cruz de Tenerife FUENTE: ELABORACIÓN PROPIA

En esta última unidad, la segunda periferia, se ubica el barrio de Añaza. El interés de este caso se fundamenta en su origen y desarrollo urbano. Ańaza es un barrio vulnerable con algo más de 9.000 residentes, surgido en la década de 1980 para realojar a personas procedentes de sectores urbanos centrales con procesos de gentrificación. Esta zona del barrio registra un cierto envejecimiento, con una edad media que supera los cuarenta ańos, hogares de tres miembros o más y carencias significativas en el estado de sus viviendas (Censo de Población y Viviendas, 2011). Desde mediados de la década de 1990, a partir de la construcción del primer gran centro comercial de la ciudad, el barrio comenzó a resultar atractivo a la inversión inmobiliaria; se construyeron bloques multifamiliares de promoción privada, coincidiendo con el periodo del boom inmobiliario. Siempre según el Censo, la población joven local de clase media y baja forma hogares más pequeños que los 
del núcleo primigenio, de dos personas como promedio, y accede a estos bloques mediante el préstamo hipotecario. De ese modo, se ha gestado un barrio con una cierta diversidad interna tanto en el plano urbanístico como en el sociodemográfico, en el que adquiere pleno sentido un análisis en la dirección que se propone. Si bien es cierto que la presencia de vivienda social hace que la tasa de ejecuciones hipotecarias sea inferior a la de otros barrios del entorno, si tomamos los valores absolutos, Añaza se sitúa entre los ámbitos con mayor número de ejecuciones, lo que pone de relieve las dificultades surgidas en el barrio a partir de la crisis y la profundización y la difusión de su vulnerabilidad social.

\section{Expansión inmobiliaria, crisis y acumulación por desposesión: análisis intraurbano de las ejecuciones hipotecarias}

A partir de mediados de la década de 1990, la orientación de España y otros países del sur de Europa hacia la inversión de capitales en el circuito secundario se tradujo, a nivel espacial, en el despliegue de un tsunami urbanizador (Fernández Durán, 2006). Con ese modelo se desencadenó una burbuja de precios sin precedentes, sostenida mediante la difusión del crédito hipotecario y el sobreendeudamiento familiar (Gutiérrez \& Declòs, 2017; López \& Rodríguez, 2011). En los últimos años del siglo xx y primeros del xxi, la producción de vivienda en Santa Cruz de Tenerife estuvo acompañada de un destacado aumento de los precios. Dos apuntes al respecto: entre 1999 y 2000 la oferta de vivienda nueva prácticamente se duplicó, pasando de 1.358 a 2.477 unidades, en un contexto en el que el aumento poblacional era moderado -213.950 habitantes en 1999 y 215.132 el año 2000- (figura 2). Desde esa fecha hasta 2006, la producción de inmuebles se mantuvo en valores elevados, aunque con algunas fluctuaciones, mientras que el incremento de precios ha sido constante, pasando de 831 a $2.428 € / \mathrm{m}^{2}$ entre 1997 y 2007 . En esta coyuntura, el crédito hipotecario ha sido la opción preferente para acceder a la vivienda, mientras que el alquiler se encarece y no cuenta con el respaldo institucional (Burriel, 2008; López \& Rodríguez, 2011). Por tanto, la financiarización de la economía se produce simultáneamente al sobreendeudamiento familiar, un modelo cuyas debilidades han aflorado con el estallido de la crisis (Rehner \& Rodríguez-Leiva, 2017). En Santa Cruz de Tenerife, se refrenda la idea del despliegue de las crisis en torno a los procesos de urbanización y de la conversión de las ciudades en el punto de colisión masiva de la acumulación por desposesión impuesta sobre los grupos menos pudientes (Harvey, 2008). En tal sentido, el examen que sigue aporta algunas claves.

En la ciudad de Santa Cruz, los contrastes entre las distintas áreas urbanas resultan esenciales en la comprensión de las lógicas espaciales de los procesos de desposesión que toman protagonismo con la crisis. La segunda periferia destaca por el peso de los procedimientos asociados al impago de la hipoteca (figura 3). De hecho, de los diez barrios con las tasas de ejecución hipotecaria más elevadas, seis se encuadran en esa unidad: Santa María del Mar (51,5\%o), Barranco Grande (22,8\%o), Alisios (21,0\%o), La Gallega (20,0\%o), Acorán (19,6\%o) y El Sobradillo $(19,5 \%$ ). La segunda periferia es el ámbito principal de crecimiento inmobiliario reciente de la ciudad, por lo que no es de extrañar que la hiperproducción de 
viviendas y la desorbitada expansión del medio construido guarden estrecha relación con la mayor intensidad de la crisis hipotecaria (Gutiérrez \& Declòs, 2017). Estas nuevas periferias se orientan de modo mayoritario al alojamiento de población joven trabajadora de bajo poder adquisitivo que accede a la vivienda a través del endeudamiento hipotecario (tabla 1). Con la crisis, el incremento del desempleo y la precariedad laboral han desembocado en dificultades para asumir el pago de las deudas contraídas y justifican la magnitud de la crisis hipotecaria, pues este sector de la ciudad se sitúa a la cabeza en la proporción de hogares con ingresos mensuales inferiores a 1000 euros y fue también el que registró un mayor incremento del desempleo entre 2009 y 2012 (IMAs, 2014). Por ello, resulta sintomático que en la segunda periferia haya casi un $80 \%$ de viviendas en propiedad y sea el sector en el que una mayor proporción de residentes (58\%) considera que los gastos de la vivienda son una carga pesada (Ibid.). Por tanto, la mayor densidad de los desalojos en los sectores urbanos más frágiles y precarios conduce a ratificar la dimensión de clase de la crisis, al tiempo que apoya los planteamientos que señalan la intensificación del desarrollo geográfico desigual en contextos de recesión (Aalbers, 2009; García, 2010; Smith, 2010).

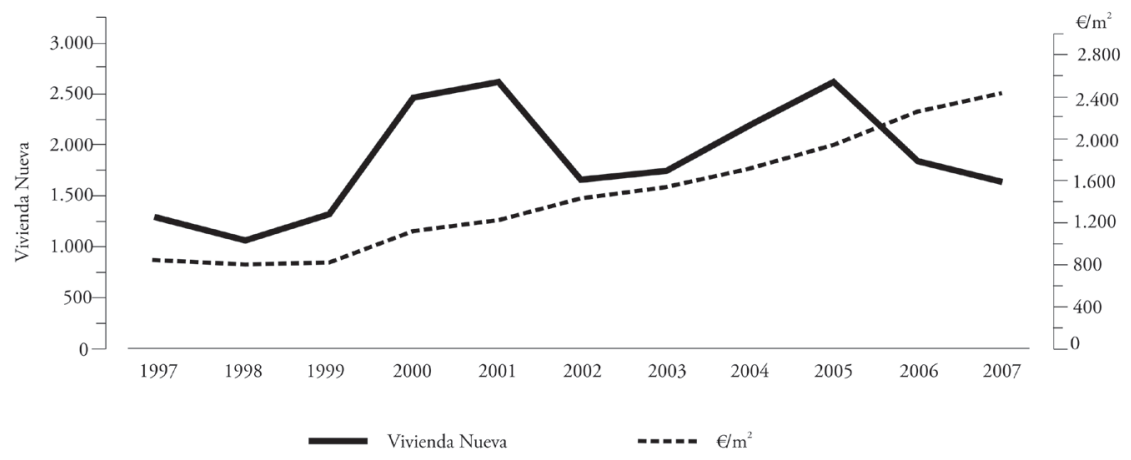

FIgura 2 | Producción y precios de la vivienda nueva en Santa Cruz de Tenerife (1997-2007)

FUENTE: ELABORACIÓN PROPIA CON BASE EN ARCAS, MUÑOZ Y CRISTÓBAL (2007)

\begin{tabular}{|l|c|c|c|c|c|c|c|}
\cline { 3 - 8 } \multicolumn{1}{c|}{} & \multicolumn{2}{c|}{} & \multicolumn{2}{c|}{ MAYORES DE 65 AÑOS } & \multicolumn{2}{c|}{ SIN ESTUDIOS } & \multicolumn{2}{c|}{ PARADOS } \\
\cline { 2 - 8 } \multicolumn{1}{c|}{} & POBLACIÓN & TOTAL & $\%$ & TOTAL & $\%$ & TOTAL & $\%$ \\
\hline Centro urbano & 63.255 & 12.585 & 19,90 & 2.935 & 4,64 & 9.180 & 14,52 \\
\hline Primera periferia & 79.680 & 15.280 & 19,18 & 7.715 & 9,68 & 16.685 & 20,94 \\
\hline Segunda periferia & 46.075 & 3.340 & 7,25 & 2.725 & 5,91 & 9.620 & 20,88 \\
\hline
\end{tabular}

TABLA I Indicadores demográficos y socioeconómicos de Santa Cruz de Tenerife (2011)

FUENTE: CENSO DE POBLACIÓN Y VIVIENDAS DE 20 I I 
Una cuestión relevante que hasta el momento ha sido poco considerada en la investigación geográfica es el desigual peso de las ejecuciones hipotecarias en distintos sectores desfavorecidos. En tal sentido, se advierte que, en los barrios de la primera periferia obrera, la incidencia de los desalojos es bastante menor (figura 3), pues la mayoría de ellos presentan tasas de ejecuciones hipotecarias muy bajas: Cuesta Piedra (1,0\%o), Finca La Multa (4,2\%o), Las Retamas (4,6\%o) y San Pío (4,8\%o). El origen y evolución de este conjunto de barrios a partir de autoconstrucción y polígonos de vivienda pública y privada anteriores a 1980 explica la reducida entidad de los procesos de desposesión de vivienda. Se trata de un sector en el que se ubica la mayor parte de las áreas vulnerables de la ciudad y que registra un elevado índice de envejecimiento, bajo nivel formativo y alta tasa de paro (tabla 1). La mayoría de sus residentes ya tiene la vivienda pagada, por lo que la exposición al crédito hipotecario es baja. Este hecho motiva a preguntarse por la forma en que los desalojos por ejecución hipotecaria intervienen en la difusión espacial de la vulnerabilidad social. Mientras los espacios desfavorecidos experimentan con la crisis una profundización de su precariedad, visible en el porcentaje de parados de la periferia y en la cifra de demandantes de asistencia social (Sociedad de Desarrollo, 2013), la desposesión de vivienda revela la propagación de las dificultades sociales entre los residentes de menor poder adquisitivo de sectores urbanos que hasta el momento no han sido reconocidos como ámbitos vulnerables.

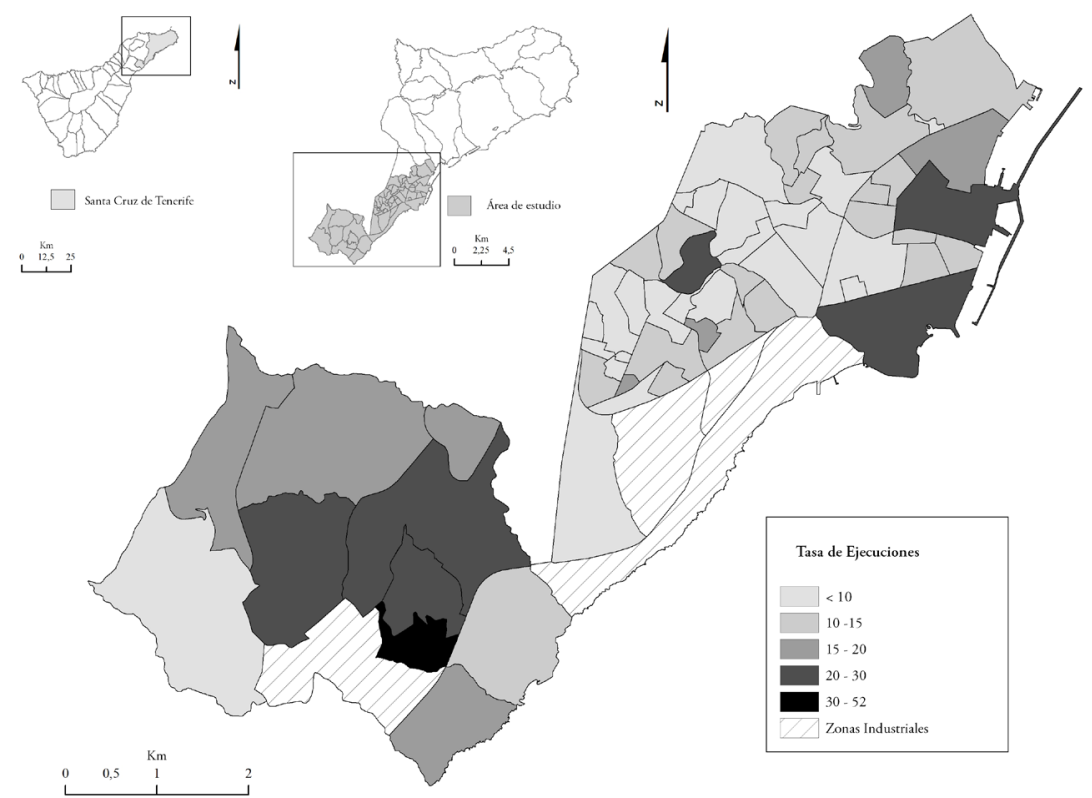

FIgUra 3 | Tasas de ejecuciones hipotecarias (\%) en Santa Cruz de Tenerife (2008-2015)

FUENTE: ELABORACIÓN PROPIA CON BASE EN DATOS ATLANTE (CGPJ) 
En esa dirección, se comprueba que incluso en algunos barrios del centro urbano se registra una incidencia significativa de las ejecuciones hipotecarias. Se trata de los espacios más céntricos y emblemáticos de la ciudad que han experimentado importantes transformaciones desde finales del siglo xx. Tales áreas, junto a las zonas que acogen la expansión metropolitana más reciente, se han convertido en los escenarios hegemónicos de la financiarización y la apropiación de las rentas urbanas (Gutiérrez \& Domènech, 2017; Vives-Miró, González, \& Rullán, 2015), A partir de la crisis, ello ha derivado en la emergencia de las ejecuciones hipotecarias como una variable clave de la profundización y la difusión de la vulnerabilidad social, al tiempo que constituye una de las manifestaciones más perversas del despliegue del urbanismo neoliberal. Además, en estas áreas de expansión reciente y de cierta calidad constructiva, los desalojos implican la acumulación de viviendas por parte de entidades financieras y fondos de inversión, que quedan a la espera de revalorizaciones futuras que les aporten nuevos beneficios (Vives-Miró, 2018).

\section{Pérdida de la vivienda y vulnerabilidad social: las realidades emergentes de la precariedad urbana en Añaza}

Las ciudades constituyen un marco espacial en el que se reflejan las contradicciones latentes entre la acumulación de capital y las necesidades que emanan desde las esferas de la reproducción social (Harvey, 2003). A partir de 2007, los procesos de desposesión evidencian la divergencia entre los intereses de los inversores y las entidades bancarias, y las dificultades de aquellas familias vulnerables que no pueden conservar su derecho a la vivienda. El análisis de las geografías de la desposesión en Añaza persigue caracterizar las áreas de alojamiento afectadas por las ejecuciones hipotecarias y reflexionar sobre la necesidad de redefinir la vulnerabilidad social en el contexto de poscrisis.

Durante el periodo 2008-2015 se registró en Añaza un elevado volumen de ejecuciones hipotecarias (103) que se convirtieron en un factor ańadido a su vulnerabilidad, al tratarse de uno de los barrios con mayor cuantía de procedimientos asociados al impago de la hipoteca. El volumen de desalojos en este barrio converge con lo que ocurre en otras ciudades del archipiélago balear y del litoral mediterráneo, en donde las ejecuciones hipotecarias se concentran, en especial, en los barrios periféricos de alojamiento de población trabajadora (Gutiérrez \& Declòs, 2017; VivesMiró, Rullán, \& González, 2018). En aquellos barrios en los que se conjuga una situación socioeconómica inestable con una alta exposición al riesgo hipotecario se produce la mayor densidad de desalojos. Gutiérrez y Domènech (2018) demuestran la correlación entre los indicadores de vulnerabilidad y las ejecuciones hipotecarias, y constatan que la crisis golpeó con más fuerza a los barrios más precarios. En Añaza, los procedimientos asociados al impago de la hipoteca comenzaron a tener presencia con el estallido de la burbuja inmobiliaria, pero fue a partir de 2012, coincidiendo con la reciente implantación de las políticas de austeridad y recortes sociales (Piñeira \& Trillo, 2016), cuando la situación se agravó, con el 75\% de las ejecuciones hipotecarias del periodo concentradas entre 2012 y 2015 (tabla 2). 


\begin{tabular}{|c|c|c|c|c|c|c|c|c|c|c|}
\hline \multirow{3}{*}{$\begin{array}{l}\text { EJECUCIONES } \\
\text { HIPOTECARIAS }\end{array}$} & \multicolumn{4}{|c|}{ VIVIENDA PROTEGIDA } & \multicolumn{4}{|c|}{ VIVIENDA LIBRE } & & \\
\hline & \multicolumn{2}{|c|}{ I990-1995 } & \multicolumn{2}{|c|}{$1996-2013$} & \multicolumn{2}{|c|}{ 1990-1995 } & \multicolumn{2}{|c|}{$1996-2013$} & \multicolumn{2}{|c|}{ TOTAL } \\
\hline & $\mathbf{N}^{\mathbf{o}}$ & $\%$ & $\mathbf{N}^{\mathbf{o}}$ & $\%$ & $\mathbf{N}^{\mathbf{o}}$ & $\%$ & $\mathbf{N}^{\mathbf{o}}$ & $\%$ & $\mathbf{N}^{\mathbf{o}}$ & $\%$ \\
\hline 2008-2011 & 7 & 6,8 & 1 & 1,0 & 12 & 11,7 & 6 & 5,8 & 26 & 25,2 \\
\hline $2012-2015$ & 7 & 6,8 & 23 & 22,3 & 13 & 12,6 & 34 & 33,0 & 77 & 74,8 \\
\hline Total & 14 & 13,6 & 24 & 23,3 & 25 & 24,3 & 40 & 38,8 & 103 & 100 \\
\hline
\end{tabular}

TABLA 2 Ejecuciones hipotecarias en Añaza según la tipología residencial

FUENTE: ELABORACIÓN PROPIA CON BASE EN PLAN GENERAL DE ORDENACIÓN DE SANTA CRUZ DE TENERIFE DE 20 I 3 Y BASE DE DATOS ATLANTE (CGPJ)

En la distribución de los desalojos en el barrio, se observa que en los bloques de vivienda libre hay un mayor predominio, en especial en los construidos a partir de 1995 (figura 4). Se trata de espacios residenciales de cierta calidad urbanística, próximos a los principales servicios y equipamientos del barrio -centro comercial, instituto, oficina de correos, centro de salud, etcétera-. A partir de la recesión, la pérdida de empleo y la precariedad laboral en estos espacios ha sido acusada y en ellos la crisis hipotecaria ha incidido con especial virulencia, debido al endeudamiento de familias jóvenes de clase media y baja que optaron por la compra de una vivienda en la fase expansiva. Por el contrario, en las viviendas protegidas el volumen de desalojos es menor, si bien su análisis en detalle revela algunas particularidades. En el núcleo primigenio del barrio de vivienda protegida, aledaño a la Rambla de Añaza y con un mayor nivel de deterioro, los desalojos son irrelevantes y solo aparece algún caso; en cambio, en los bloques más recientes y mejor situados el fenómeno empieza a ser reseńable a partir de 2012. Se registra en ellos casi una cuarta parte del total de ejecuciones hipotecarias (tabla 2). A falta de un análisis en profundidad sobre ese particular, el paso de las viviendas a propiedad tiempo después de ser ocupadas hace que aquellas insertas en la zona mejor valorada del barrio hayan sido objeto preferente de operaciones de compraventa. En Añaza, los discursos de los residentes acerca de la dimensión urbanística del barrio expresan con claridad las diferencias entre las deficientes condiciones de habitabilidad y mantenimiento de la zona tradicional de vivienda protegida y el resto del barrio donde predomina la vivienda libre de mayor calidad constructiva, que se combina con algunos bloques de protección oficial con un mejor grado de conservación.

Yo pienso que sí hay diferencias, claramente [...]. Las vpo [Viviendas de Protección Oficial] de toda la parte social de arriba están destrozadas. Si tú paseas por dentro, las escaleras rotas, están las vallas tiradas, los jardines rotos [...]. Qué pasa, que hay problemas en eso, pues estas casas pasaron a propiedad después de tantos años y como no están formadas las comunidades de vecinos, el mantenimiento no se hace. (Vecina desde 1989, 33 años)

Un discurso antagónico es el planteado por aquellos que residen en la zona mejor valorada del barrio, en espacios con un menor deterioro físico en los que las ventajas en la dimensión urbanística -calles amplias, proximidad a los servicios y 
equipamientos del barrio, buen estado de las viviendas- encubren la existencia de dificultades socioeconómicas tras la crisis y, también, de otras debilidades, como la ausencia de vida urbana y lazos comunitarios.

Estuvimos visitando varias zonas de Santa Cruz y preferimos vivir no en el centro de la ciudad, sino en la periferia... Encontramos Ańaza, como decir, indagando, caminando, y nos enamoramos de Añaza; son calles anchas, lo vimos bastante ordenado... Una vecina gentilmente nos enseñó su apartamento y nos encantó; está bien distribuido, tiene su plaza de parking, trasteros, solana, dos habitaciones, dos baños. Para mi señora y para mí estaba fenomenal. (Vecino desde 2008, 63 años)

En definitiva, la mayor densidad de los desalojos en el sector del barrio con mejor calidad urbanística parece poner en cuestión la habitual vinculación de la vulnerabilidad social con la espacial, es decir, con aquellas áreas de la ciudad donde el elevado grado de deterioro físico es la expresión de situaciones sociales complicadas. El análisis de caso conduce a plantear el papel de los desalojos en la extensión de la precariedad desde los ámbitos más dañados de vivienda protegida, surgidos para el alojamiento de personas en riesgo de pobreza, hacia las áreas de desarrollo más reciente, destinadas al alojamiento de población joven de clase trabajadora que ha adquirido la vivienda vía crédito hipotecario. Se evidencia, por tanto, que la crisis de 2007, además de acentuar las dificultades sociales de los grupos más vulnerables, empuja también a situaciones adversas a residentes de clases medias y bajas que, en un momento de pérdida de empleo y de reducción de los ingresos familiares, tienen serias dificultades para seguir pagando la hipoteca.

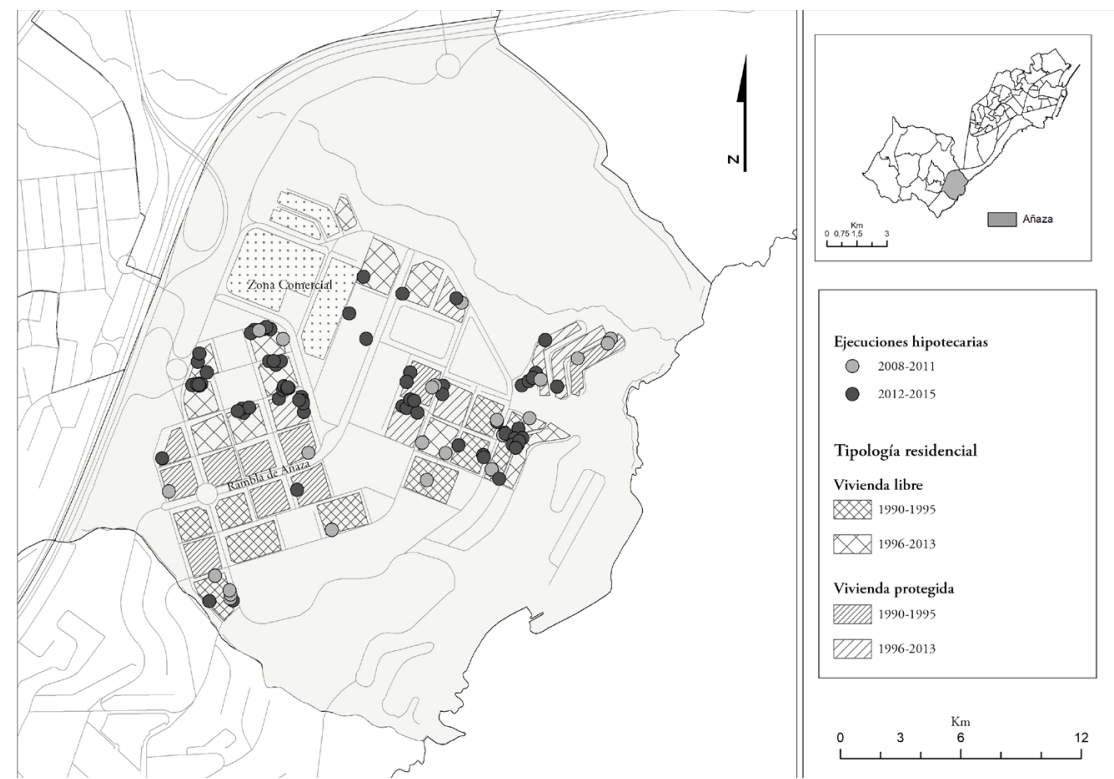

FIGURA 4 | Ejecuciones hipotecarias según la tipología residencial en el barrio de Ańaza (2008-2015)

FUENTE: ELABORACIÓN PROPIA CON BASE EN PLAN GENERAL DE ORDENACIÓN DE SANTA CRUZ DE TENERIFE DE 2013 Y BASE DE DATOS ATLANTE (CGPJ) 
Desde la geografía se ha prestado escasa atención a cualificar los espacios urbanos en los que la desposesión de vivienda adquiere magnitudes destacadas. La aproximación cualitativa a estos lugares se postula como un modo de análisis relevante para comprender las realidades emergentes de la existencia cotidiana en la ciudad neoliberal. Siguiendo el planteamiento de Bourdieu (1990), cuando un grupo de individuos ocupa posiciones semejantes en el espacio social y está sometido a similares condiciones en su vida diaria, tiene todas las probabilidades de producir prácticas, posiciones y decisiones semejantes. En Ańaza se pueden identificar comportamientos diferenciados, e incluso opuestos, entre los residentes de la zona de vivienda protegida y los que habitan en las promociones de vivienda libre situadas en las áreas de nuevo crecimiento del barrio y afectadas en mayor medida por los desalojos. Entre los primeros, el desarrollo de un enérgico movimiento vecinal ha sido un factor clave en la incorporación de los servicios y equipamientos sociales con los que se ha dotado al barrio (García-Hernández, 2018). Esa reacción de los residentes, articulando mecanismos para la mejora de sus condiciones de vida, muestra rasgos similares a luchas ciudadanas que caracterizaron al país durante el periodo de transición política (Recio \& Naya, 2004; Urrutia, 1992).

Desde luego, si no hubiese sido por las luchas vecinales, eso [el barrio] no sería lo que ahora es, en absoluto. Nada existiría, nada. (Vecino desde 1989, 65 ańos)

El desarrollo de un tejido social sólido se traduce también en el despliegue de lazos de vecindad y solidaridad entre los residentes y repercute en el despliegue de sentimientos de identificación y pertenencia hacia sus lugares de vida diaria, especialmente entre los residentes más jóvenes.

Sí, yo creo que sí. Como ya lleva más de veinte años el barrio, aquí la gente se conoce; y sí, esto es como una gran familia. (Vecina desde 1993, 26 años)

A estas dinámicas permanecen ajenos los habitantes que se incorporaron al barrio a partir de la expansión inmobiliaria reciente. La satisfacción de las demandas más acuciantes con anterioridad a su llegada parece explicar su escasa implicación en los colectivos vecinales.

No mucho. Mucha gente vive independiente. No sé hasta qué punto se integran todos; más bien donde está la comunidad de vecinos, pero del resto, por aquí no veo que se integren mucho. (Vecino desde 2008, 60 años)

En definitiva, a partir de la crisis hipotecaria, los procesos de desposesión de viviendas se han convertido en una variable esencial para la redefinición de las desigualdades intraurbanas (Gutiérrez \& Declòs, 2017; Vives-Miró, González, \& Rullán, 2015). En un barrio como Añaza, la distribución espacial de los desalojos revela la propagación de las dificultades sociales entre aquellos que en la etapa precrisis adquirieron una vivienda contratando una hipoteca, residentes en ámbitos de cierta calidad urbanística y cuyo perfil no encaja con los cánones convencionales de la vulnerabilidad. La tendencia al individualismo y la ausencia de dinámicas comunes de participación, habitual en estos sectores urbanos (Bauman, 2001; Hackworth, 2013), debe ser sustituida por prácticas vecinales que aboguen, entre otras necesidades, por el derecho a la vivienda. 


\section{Reflexiones finales}

En el marco interpretativo del derecho a la ciudad, la vivienda es un bien imprescindible para la ciudadanía y escenario central de la existencia cotidiana. En la ciudad neoliberal, la administración elude la responsabilidad de facilitar su acceso a los colectivos vulnerables y de rectificar la condición del suelo y de la vivienda como mercancías sujetas a los vaivenes de los ciclos económicos. En Santa Cruz de Tenerife, como en otras ciudades españolas, se comprueba la disonancia entre los elevados niveles de producción de viviendas, el ritmo del crecimiento poblacional y el incremento continuado de los precios en la fase del urbanismo expansivo. La generalización del crédito hipotecario explica el acceso a la vivienda y se halla en la raíz de la proliferación de los procesos de desposesión a partir de 2007.

El papel de las ejecuciones hipotecarias en la difusión espacial de la vulnerabilidad social se puede inferir del análisis intraurbano y del estudio en detalle a escala de barrio. En la primera periferia urbana se comprueba la escasa entidad de los desalojos debido a su origen y consolidación anterior a la década de 1980, a la concentración de buena parte de la vivienda social de la ciudad y a la ausencia de procesos recientes de revalorización inmobiliaria. Por el contrario, en aquellos sectores del centro urbano en los que la burbuja de precios ha sido potente, el peso de las ejecuciones es superior, y aún más en la segunda periferia, donde la disponibilidad de suelo se tradujo en la ampliación del parque inmobiliario durante la etapa precrisis y en la expansión del crédito hipotecario. Su condición de áreas de residencia de población trabajadora confirma que la crisis afecta en mayor medida a aquellas familias de bajo poder adquisitivo $y$, por tanto, que contribuye a reforzar la desigualdad de clase.

El caso de Añaza permite destacar el papel de los desalojos en la extensión de la vulnerabilidad. La geografía de las ejecuciones hipotecarias confirma que las dificultades sociales no se concentran en exclusiva en las zonas más degradadas de vivienda protegida, sino que se extienden hacia sectores del barrio de mayor calidad urbanística, cuyos residentes, en contextos de inestabilidad laboral y pérdida de empleo, no pueden asumir el pago de la hipoteca. La investigación cualitativa revela que estos espacios producidos bajo las pautas del expansionismo neoliberal ofrecen una serie de particularidades que refuerzan su vulnerabilidad. La fragilidad de las estrategias colectivas de lucha ciudadana y de los lazos vecinales es un obstáculo ańadido en sectores urbanos con un menor deterioro físico-urbanístico, pero donde existen diversos problemas menos visibles, que se deben tener en cuenta para una adecuada interpretación de la existencia cotidiana en esos espacios.

En definitiva, el despliegue de los proyectos de reestructuración neoliberal genera escenarios urbanos sujetos a los efectos de las crisis periódicas y caracterizados por la dominación y el control ejercido por las elites financieras y los grupos de poder sobre los colectivos vulnerables. Los espacios destinados a la reproducción social constituyen un ámbito idóneo para la comprensión de las múltiples contradicciones que resultan del modo imperante de producción del espacio urbano, pues en ellos se materializan las prácticas que despojan a los ciudadanos de las posibilidades para conseguir unas condiciones adecuadas para la vida cotidiana. Por tanto, 
la persistencia en los postulados neoliberales, unida a la ausencia de estrategias de reapropiación del espacio, se traducen en la configuración de unas geografías urbanas cada vez más desiguales.

\section{Agradecimientos}

Los autores agradecen las aportaciones de los revisores y las mejoras sugeridas a la versión previa de este artículo. El presente trabajo forma parte del proyecto de investigación del Plan Nacional de I+D+I "Crisis y vulnerabilidad en ciudades insulares espańolas. Transformaciones en los espacios de reproducción social” (CSO2O I 5 68738-P), Ministerio de Economía y Competitividad (MIneco)-Programa Retos (Aei/feder, Ue), financiado por el mineco. Trabajo cofinanciado por la Agencia Canaria de Investigación, Innovación y Sociedad de la Información de la Consejería de Economía, Industria, Comercio y Conocimiento y por el Fondo Social Europeo (FSE), Programa Operativo Integrado de Canarias 2014-2020, Eje 3, Tema Prioritario 74 (85\%).

\section{Referencias bibliográficas}

Aalbers, M. (2009). Geographies of financial crisis. Area, 41(1), 34-42. https://doi.org/10.1111/ j.1475-4762.2008.00877.x

Arcas, I. Muñoz, J., \& Cristóbal, A. (coords.) (2007). Estudio de oferta de vivienda de nueva construcción en España. Año 2007. Ministerio de Vivienda/GfK. https://bit. ly/30CNZnS

Armas, A. (2016). Reestructuración urbana y producción de imagen: los espacios públicos en Santa Cruz de Tenerife. Tesis doctoral inédita. Universidad de La Laguna. https://riull.ull.es/ xmlui/handle/915/2126

Ayuntamiento de Santa Cruz de Tenerife (2013). Plan General de Ordenación de Santa Cruz de Tenerife. Documento de Aprobación Definitiva Parcial. https://www. santacruzdetenerife.es/servicios-municipales/urbanismo/plan-general-de-ordenacionde-santa-cruz-de-tenerife-pgo/

Bauman, Z. (2001). La sociedad individualizada. Madrid: Cátedra.

Bourdieu, P. (1990). Sociología y cultura. México DF: Grijalbo.

Brenner, N. \& Theodore, N. (2002) (eds.). Spaces of neoliberalism: urban restructuring in North America and Western Europe. Malden, Ma: Blackwell Publishing.

Bülher, E., Kaspar, H., \& Ostermann, F. (2010). Sozialenachhaltilige Parkanlagen. Zúrich: Forchunsberich des Nationales Forschungsprogramms.

Burriel, E. (2008). La "década prodigiosa" del urbanismo español (1997-2006). Scripta Nova. Revista Electrónica de Geografía y Ciencias Sociales, 12(270). http://www.ub.edu/ geocrit/-xcol/383.htm

Burriel, E. (2016). Empty urbanism: the bursting of the Spanish housing bubble. Urban Research \& Practice, 9(2), 158-180. http://roderic.uv.es/handle/10550/57166 
Carlos, A. (2007). O espaço urbano: Novos escritos sobre a ciudade. São Paulo: Faculdade de Filosofia, Letras e Ciências Humanas da Universidade de São Paulo (FFlCH-usp). http://www.gesp.fflch.usp.br/sites/gesp.fflch.usp.br/files/Espaco_urbano.pdf

Charnock, G., Purcell, T. F., \& Rivera-Fumaz, R. (2015). The limits to capital in Spain: the roots of the 'new normal'. Critique: Journal of Socialist Theory, 43(2), 173-188. https:// doi.org10.1080/03017605.2015.1051785

Coq-Huelva, D. (2013). Urbanisation and financialisation in the context of a rescaling state: the case of Spain. Antipode, 45(5), 1213-1231. https://doi.org/10.1111/anti.12011

Díaz, M. C., Delgado, C. R., Calero, C. G., García, L. M., Pérez, M., \& Mejías, M. A. (2002). Ciudad oscura, ciudad luminosa. Estudios Geográficos, (28), 33-51. https:// doi.org10.14198/INGEO2002.28.06

Fernández Durán, R. (2006). El tsunami urbanizador español y mundial: sobre sus causas y repercusiones devastadoras y la necesidad de prepararse para el previsible estallido de la burbuja inmobiliaria. Barcelona: Virus Editorial.

García, M. (2010). The breakdown of the Spanish urban growth model: social and territorial effects of the global crisis. International Journal of Urban and Regional Research, 34(4), 967-980. https://doi.org/10.1111/j.1468-2427.2010.01015.x

García-Hernández, J. S. (2017). El espacio público en periferias desfavorecidas: Añaza y Santa Clara paradigmas de vulnerabilidad socioespacial en Santa Cruz de Tenerife. Scripta Nova. Revista Electrónica de Geografía y Ciencias Sociales, 21(571), 1-32. http://dx.doi. org/10.1344/sn2017.21.19719

García-Hernández, J. S. (2018). La transformación de la cotidianidad urbana en los espacios de reproducción social: movimientos vecinales en barrios desfavorecidos de Santa Cruz de Tenerife (España). Boletin de la Asociación de Geógrafos Españoles, 79(2442), 1-27. http://dx.doi.org/10.21138/bage.2442

García-Hernández, J. S., Díaz-Rodríguez, M. C., \& García-Herrera, L. M. (2018). Auge y crisis inmobiliaria en Canarias: desposesión de vivienda y resurgimiento inmobiliario. Investigaciones Geográficas, (69), 23-39. https://doi.org/10.14198/INGEO2018.69.02

García-Herrera, L. M., Smith, N., \& Mejías, M. A. (2007). Gentrification, displacement and tourism in Santa Cruz de Tenerife. Urban Geography, 28(3), 276-298. https://doi. $\operatorname{org} 10.2747 / / 0272-3638.28 .3 .276$

Gutiérrez, A. \& Declòs, X. (2017). Geografía de la crisis inmobiliaria en Cataluña: una lectura a partir de los desahucios por ejecución hipotecaria. Scripta Nova. Revista Electrónica de Geografía y Ciencias Sociales, 21(557). http://revistes.ub.edu/index.php/ScriptaNova/ article/view/17734

Gutiérrez, A. \& Domènech, A. (2017). Spanish mortgage crisis and accumulation of foreclosed housing by SAREB: a geographical approach. Journal of maps, 13(1), 130-137. https:// doi.org/10.1080/17445647.2017.1407271

Gutiérrez, A. \& Domènech, A. (2018). The mortgage crisis and evictions in Barcelona: identifying the determinants of the spatial clustering of foreclosures. European Planning Studies, 26(10), 1939-1960. https://doi.org/10.1080/09654313.2018.1509945

Gutiérrez, A. \& Vives-Miró, S. (2018). Acumulación de viviendas por parte de los bancos a través de los desahucios: geografía de la desposesión de vivienda en Cataluña. EURE, 44(132), 5-26. http://dx.doi.org/10.4067/s0250-71612018000200005 
Hackworth, J. (2013). The neoliberal city. Governance, ideology and development in American Urbanism. Ithaca, NY: Cornell University Press.

Harvey, D. (2003). The new imperialism. Oxford, uk / Nueva York: Oxford University Press.

Harvey, D. (2007). Espacios del capital. Madrid: Akal.

Hay, I. (2010). Qualitative research methods in human geography. Oxford, uk: Oxford University Press.

Human Right Watch (2014). Sueños rotos. El impacto de la crisis española de la vivienda en grupos vulnerables. New York: Human Right Watch.

Instituto Municipal de Atención Social (2014). Diagnóstico Social del Municipio de Santa Cruz de Tenerife. I Plan Municipal de Prevención Social y Desarrollo Comunitario. Santa Cruz de Tenerife: Ayuntamiento de Santa Cruz de Tenerife.

Iveson, K. (2007). Publics and the city. Oxford, uk: Blackwell Publishing.

Lefebvre, H. (1974). La Production de l'espace. París: Anthropos.

Lefebvre, H. (1969). El derecho a la ciudad. Barcelona: Ediciones Península.

Lois, R., Piñeira, M. J., \& Vives, S. (2016). El proceso urbanizador en España (1990-2014): una interpretación desde la geografía y la teoría de los circuitos de capital. Scripta Nova. Revista Electrónica de Geografia y Ciencias Sociales, 20(539), 1-29. http://dx.doi. org/10.1344/sn2016.20.16793

López, I. \& Rodríguez, E. (2011). The Spanish model. New Left Review, 69, 5-28. https:// newleftreview.org/issues/II69

Mayring, P. (2000). Qualitative Inhaltsanalyse. Forum Qualitative Social Research, 1(2). http:// www.qualitative-research.net/index.php/fqs/article/view/1089/2383

Méndez, R. \& Plaza, J. (2016). Crisis inmobiliaria y desahucios hipotecarios en España: una perspectiva geográfica. Boletín de la Asociación de Geógrafos Españoles, (71), 99-127. http://dx.doi.org/10.21138/bage.2276

Ministerio de Fomento (2011). Atlas de la Vulnerabilidad Urbana en España. Gobierno de España. Portal de Suelo y Políticas Urbanas. http://atlasvulnerabilidadurbana.fomento. es/\#1=es; $=$ map2

Naredo, J. M. (2010). El modelo inmobiliario español y sus consecuencias. Boletín $C F+S$, (44), 13-27. http://habitat.aq.upm.es/boletin/n44/ajnar.html

Parreño-Castellano, J. M., Domínguez-Mujica, J., Armengol-Martín, M., Pérez, T., \& Boldú, J. (2018). Foreclosures and evictions in Las Palmas de Gran Canaria during the economic crisis and post-crisis period in Spain. Urban Science, 2(109), 1-15. https:// doi.org/10.3390/urbansci2040109

Piñeira, M. J. \& Trillo, J. M. (2016). El efecto de la crisis sobre los barrios vulnerables: una aproximación al contexto español a través de un estudio de caso. En Crisis, globalización $y$ desequilibrios sociales y territoriales en España. Aportación Española al 33 Congreso de la Unión Geográfica Internacional. Beijing. http://www.age-geografia.es/site/wp-content/ uploads/2016/07/crisis_globlalizacion_UGI_spa_2016_WEB.pdf

Obeso, I. (2014). Análisis geográfico de los desahucios en España. Eria, (95), 327-342. https:// www.unioviedo.es/reunido/index.php/RCG/article/view/10513

Recio, A. \& Naya, A. (2004). Movimiento vecinal: claroscuros de una lucha necesaria. Mientras Tanto, (91/92), 63-81.

Smith, N. (2010). Uneven development: Nature, capital, and the production of space. Athens, GA: University of Georgia Press. 
Sociedad de Desarrollo. (2013). Santa Cruz de Tenerife, situación socioeconómica y crisis. Datos actualizados a mayo de 2013. Santa Cruz de Tenerife: Observatorio Socioeconómico de la Sociedad de Desarrollo.

Taylor, S.H. \& Bogdan, R. (1996). Introducción a los métodos cualitativos de investigación. La búsqueda de significados. Barcelona: Paidós.

Theodore, N., Peck, J., \& Brenner, N. (2009). Urbanismo neoliberal: la ciudad y el imperio de los mercados. Temas Sociales, (66), 1-12 [Santiago de Chile: Ediciones SUR]. http:// www.sitiosur.cl/r.php?id=898

Urrutia, V. (1992). Transformación y persistencia de los movimientos sociales urbanos. Politica y Sociedad, 10, 49-56. http://revistas.ucm.es/index.php/POSO/article/view/ POSO9292120049A

Vinuesa, J. (2013). El festín de la vivienda. Auge y caída del negocio inmobiliario en España. Madrid: Díaz \& Pons.

Vives-Miró, S., González, J. M., \& Rullán, O. (2015). Home dispossession: the uneven geography of evictions in Palma (Majorca). Die Erde, 146(2-3), 113-126. http://www. die-erde.org/index.php/die-erde/article/view/211

Vives-Miró, S. \& Gutiérrez, A. (2017). Extracting rents through foreclosures: the rescue of Catalunya Banc as a new urban strategy following the burst of the Spanish bubble. Miscellanea Geographica, 21(4), 151-159. https://doi.org/10.1080/09654313.2018.1 515180

Vives-Miró, S. (2018). New rent seeking strategies in housing in Spain after the bubble burst. European Planning Studies, 26(10), 1920-1938. https://doi.org/10.1080/09654313.2 018.1515180

Vives-Miró, S., Rullán, O., \& González, J. M. (2018). Geografies de la despossessió d 'habitatge a través de la crisi. Els desnonaments Marca Palma. Barcelona: Icaria Editorial.

Winchester, H. P. M. \& Rofe, M. W. (2010). Qualitative research and its place in Human Geography. En Hay, I. (ed.), Qualitative research methods in human geography (pp. 3-24). Oxford, uk: Oxford University Press. 